\title{
Clostridium difficile infection in surgical patients (literature review)
}

Bogdan SOCEA ${ }^{1,2}$, Oana HALAU ${ }^{1}$, Camelia DIACONU ${ }^{3,4}$, Ovidiu Gabriel BRATU ${ }^{5,6}$, Tiberiu Paul NEAGU7,8, Mihai DIMITRIU ${ }^{9,10}$, Vlad Denis CONSTANTIN ${ }^{1,2}$

${ }^{1}$ Department of General Surgery, Emergency Clinical Hospital "Sf. Pantelimon", Bucharest, Romania ${ }^{2}$ Clinical Department No. 10, "Carol Davila" University of Medicine and Pharmacy, Bucharest, Romania ${ }^{3}$ Department of Internal Medicine, Clinical Emergency Hospital of Bucharest, Romania ${ }^{4}$ Clinical Department No. 5, "Carol Davila" University of Medicine and Pharmacy, Bucharest, Romania ${ }^{5}$ Department of Urology, "Dr. Carol Davila" Central Military Emergency University Hospital, Bucharest, Romania

${ }^{6}$ Clinical Department No. 3, "Carol Davila" University of Medicine and Pharmacy, Bucharest, Romania

${ }^{7}$ Department of Plastic Surgery and Reconstructive Microsurgery, Emergency Clinical Hospital of Bucharest, Romania

${ }^{8}$ Clinical Department No. 11, "Carol Davila" University of Medicine and Pharmacy, Bucharest, Romania

${ }^{9}$ Department of Obstetrics and Gynecology, Emergency Clinical Hospital "Sf. Pantelimon", Bucharest, Romania

${ }^{10}$ Clinical Department No. 13, "Carol Davila" University of Medicine and Pharmacy, Bucharest, Romania

\begin{abstract}
Clostridium difficile infection (CDI) has become a global health challenge, due to the dramatic worldwide increase in incidence and severity in the past three decades. Furthermore, the incidence of CDI has increased in surgical patients. The surgery itself is a known risk factor, that, together with the postoperative care (hospitalization, antibiotic treatment, intensive care unit admission), facilitate the development of CDI. Therefore, a matter of paramount importance is the optimization of the management of surgical patients and prevention of Clostridium difficile infection.
\end{abstract}

Keywords: Clostridium difficile, infection, surgery

\section{INTRODUCTION}

Clostridium difficile is an anaerobic, Grampositive, spore-forming bacillus responsible for the majority of cases of antibiotic-associated diarrhea in surgical patients. The spectrum of disease may range from asymptomatic carrier to fulminant, life-threating toxic megacolon. The typical presentation is diarrhea, which may occur at the beginning of the antibiotic therapy, including the perioperative dose. Diarrhea may be accompanied by abdominal pain, fever, and nausea $(1,2)$.

\section{Pathogenesis}

Clostridium difficile spores survive the acid environment of the stomach and germinate in 
the intestines (3). The spores act as an environmental repertoire for the bacterium and can facilitate the spread among patients, as well as contributing to the high recurrence rates observed in CDI. The primary toxins produced by the bacteria are toxin $A$ and $B$, that act as glucosyltransferases, promoting the activation of Rho GTP-ases, leading to disorganization of the cytoskeleton of the colonocyte, and eventually cell death (4). In addition to the two toxins $A$ and $B$, some strains produce a third toxin, known as binary toxin.

\section{Risk factors}

There are three general categories of risk factors: host factor, which refer to age (over 65 years), co-morbidities, immune status, inflammatory bowel diseases; exposure to Clostridium difficile spores - community sources, hospitalization; factors that disrupt normal colonic microbiome, such as surgery and antibiotics.

The disruption of the intestinal flora provides the perfect setting for proliferation and toxin production of $C$. difficile spores. Although almost all antibiotics have been associated with CDI, third-generation cephalosporins, clindamycin, fluoroquinolones and penicillin are considered to pose the greatest risk (5). It is well known that combinations of antibiotics can alter the gastrointestinal bacterial community structure and it can lead to antibiotic-associated diarrhea, including a marked reduction of many butyrate-producing bacterial members of the Clostridiaceae family, essential for colonic health. Antibiotics, by altering the intestinal microbial communities, affect the intestinal metabolome, meaning the total number of metabolites in the intestine, playing an important role in fatty acid, steroid, sugar, amino acid, bile acid and eicosanoid metabolism (5).

There is a link between CDI and surgical interventions. Recent reports have demonstrated that elderly and immunocompromised patients undergoing surgical interventions are most likely under treatment with widespread broadspectrum antibiotics, explaining the increasing incidence of $C$. difficile infection during the last decades (6).

Diarrhea is one of the most common symptoms among Intensive Care Unit (ICU) patients; the majority of cases are noninfectious and associated with complications of enteral feeding. According to recent studies, at least one out of ten patients with diarrhea are diagnosed with CDI. Clostridium difficile infection has a particular impact on ICU patients, leading to a higher rate of mortality; nevertheless, these patients have underlying conditions, more comorbidities and need more intensive care (7).

\section{Infection prevention and control}

To control an infection, it is necessary to have a strategy to control the infection outbreaks. The strategy should include a bundle approach including antibiotic stewardship, isolation measures, environmental disinfection and as simple as hand hygiene of the medical staff.

Antibiotic stewardship refers to choosing an appropriate antibiotic in optimized dose and duration, that will prevent or cure CDI. Recently, it has been proved that administration of metronidazole a day before surgery has reduced the risk of infection with Clostridium difficile in the postoperative period. A significant expected impact on the incidence of $C D I$ is the reduction of cephalosporins and quinolones, an important target for stewardship programs.

C. difficile carriers should be isolated, even if the patient is asymptomatic, proper precautions should be taken even before diagnostic laboratory test confirmation. Contact precautions in CDI will be maintained until the resolution of diarrhea, which is demonstrated by the passage of formed stool for at least 48h (8).

The keystone of the prevention of $C D I$ is hand hygiene with water and soap. The European Society of Clinical Microbiology and Infectious Diseases (ESCMID) study group for C. difficile (ESGCD) published a set of guidelines regarding measures for prevention of $\mathrm{C}$. difficile: to use a personal protective equipment (gloves and gowns); to introduce daily environmental sporicidal disinfection and terminal disinfection of rooms of patients with CDI; education of the healthcare workers on prevention of $C D I$ to enhance their knowledge and skills on prevention strategies (9).

The diagnosis of $C$. difficile infection should be based on clinical signs and symptoms, in combination with laboratory tests. It is recommended to perform stool testing only on diarrhea stools from at-risk patients: patients that had more or at least 3 loose stools in $24 \mathrm{~h}$, with no obvious alternative explication. An acceptable alternative to stool specimen analysis, in case of patients with ileus who are unable to produce a specimen, are polymerase chain reaction testing of perirectal swabs (9). In addition to the laboratory testing, the patient can take a 
series of imaging testing: CT scan in Clostridium difficile colitis, endoscopy - it can deliver a rapid diagnosis if pseudomembranes are visualized. Nevertheless, the use of endoscopy is rarely recommended in the setting of colitis, because of the high risk of perforation (10).

\section{Antibiotic therapy}

First of all, when CDI is suspected, all unnecessary antibiotic agents will be discontinued. In mild-to-moderate cases, it is believed that oral metronidazole $500 \mathrm{mg}$ three times per day for 10 days may be a successful treatment. Although metronidazole may be associated with more frequent side effects, in patients with an initial episode of non-severe CDI it is preferred, when access to vancomycin or fidaxomicin is limited (11-12). However, recent data have suggested an overall superiority of vancomycin to metronidazole for treatment of patients with CDI. Oral vancomycin $125 \mathrm{mg}$ four times a day for 10 days is recommended as first choice also for moderate cases (9).

In patients in whom oral antibiotics cannot be administrated, it is not recommended the intravenous administration. Intravenous vancomycin has no effect on CDI since the antibiotic is not excreted into the colon. A good alternative is vancomycin enema, especially for patients who cannot tolerate oral preparation - patients with ileus (12). In surgical patients with Hartmann resection, ileostomy, trans-stoma vancomycin may also be effective.

A valid alternative to vancomycin may be fidaxomicin administrated $200 \mathrm{mg}$ twice a day for a period of 10 days. Recent data showed that fidaxomicin is useful for patients who are considered at high risk of recurrence.
Patients with severe $C$. difficile infection, fulminant colitis, that progresses to systemic toxicity, and patients with organ failure require early surgical intervention. Nevertheless, emergency surgery for fulminant colitis will increase the mortality; bad prognostic factors are prolonged preoperative intubation, acute renal failure, multiple organ failure (13). In these cases, before surgery, patients with fulminant colitis should be treated with high doses of vancomycin, oral and enema, in combination with intravenous metronidazole.

An effective option for patients with multiple recurrences of $\mathrm{CDI}$ with failure of antibiotic treatment, may be the fecal microbiota transplant (FMT). Although fecal microbiota transplant has great success rates with long term durability, some disadvantages still exist (14): the laborious manipulation of feces and the enteral administration, the big costs of preparing the samples, making this treatment likely unattractive for both patients and physicians. The encapsulated preparation of FMT is a less invasive and also improved concept, that recently has had success.

\section{CONCLUSIONS}

Clostridium difficile infection has become a global public challenge, do to the increase of incidence worldwide, esspecially among surgical patients. Therefore, a better management of the disease by optimization of the perioperative treatment, postoperative care, immediate diagnosis of CDI and implementation of infection prevention and control strategies are of greatest importance in controlling this challenging infection.

Conflict of interest: none declared Financial support: none declared

\section{REFERENCES}

1. Hermsen JL, Dobrescu C, Kudsk KA. Clostridium difficile infection: A surgical disease in evolution. $J$ Gastrointest Surg 2008;12(9):1512-7.

2. McFarland VL. Update on the changing epidemiology of Clostridium difficileassociated disease. Nat Clin Pract Gastroenterol Hepatol 2008;5(1):40-8.

3. Shaughnessy MK, Micielli RL, DePestel DD, Arndt J, Strachan CL, Welch KB, Cheoweth $\mathrm{CE}$. Evolution of hospital assignment and acquisition of Clostridium difficile infection. Infect Control Hosp Epidemiol 2011;32(3):201-6.

4. Jank T, GiesemannT, Aktories K. Rhoglucosylating Clostridium difficile toxin $\mathrm{A}$ and $B$ : new insights into structure and function. Glycobiology 2007;17(4):15R-22R.

5. Young VB, Schmidt TM. Antibioticassociated diarrhea accompanied by large-scale alterations in the composition of the fecal microbiota. J Clin Microbiol 2004;42(3):1203-6.
6. Zerey M, Paton BL, Lincourt AE, Gersin KS, Kercher KW, Heniford BT. The burden of Clostridium difficile in surgical patients in the United States. Surg Infect (Larchmt) 2007;8(6):557-66.

7. Zahar JR, Schwebel C, Adrie C, GarrousteOrgeas $M$, Francais A, Vesin A, NguileMakao M, Tabah A, Laupland K, LeMonnier A, Timsit JF. Outcome of the ICU patients with Clostridium difficile infection, Crit Care. 2012;16(6):R215. 
8. Longtin Y, Paquet-Bolduc B, Gilca R, Garenc C, Fortin E, Longtin J et al. Effect of detecting and isolating Clostridium difficile carriers at hospital admission on the incidence of $\mathrm{C}$. difficile infections: a quasi-experimental controlled study. JAMA Intern Med 2016; 176(6):796-804.

9. Massiomo Sartelli, Stefano Di Bella, Lynne V. McFarland, et al. 2019 update of the WSES guidelines for management of Clostridioides (Clostridium) difficile infection in surgical patients. World Journal of Emergency Surgery 2019;14:8.
10. Hookman P, Barkin JS. Clostridium difficile associated infection, diarrhea and colitis. World J Gastroenterol 2009;15:1554-1580.

11. Nelson RL, Suda KJ, Evans CT. Antibiotic treatment for Clostridium difficile-associated diarrhoea in adults. Cochrane Database Syst Rev. 2017; 3:CD004610.

12. McDonald LC, Gerding DN, Johnson S, Bakken JS, Carroll KC, Coffin SE, et al. Clinical practice guidelines for Clostridium difficile infection in adults and children: 2017 update by the Infectious Diseases Society of America (IDSA) and Society for
Healthcare Epidemiology of America (SHEA). Clin Infect Dis 2018;66(7):e1-e48. 13. Bhangu A, Nepogodiev D, Gupta A, Torrance A, Singh P. West midlands research collaborative. Systematic review and meta-analysis of outcomes following emergency surgery for Clostridium difficile colitis. Br J Surg 2012;99(11):1501-13.

14. Orenstein R, Dubberke E, Hardi R, Ray A, Mullane K, Pardi DS, et al. Safety and durability of RBX2660 (microbiota suspension) for recurrent Clostridium difficile infection: Results of the PUNCH CD study. Clin Infect Dis 2016;62(5):596-602. 